\title{
Can Sound Public Health Policies Stem the Tide of Burgeoning Epidemic of Cardiovascular Disease in South Asians?
}

\author{
Pirbhat Shams ${ }^{1} \cdot$ Mohsina Hussain $^{2} \cdot$ Salima Karani $^{1} \cdot$ Sana Mahmood $^{3} \cdot$ Alina Hasan $^{2} \cdot$ Sameen Siddiqi $^{4}$. \\ Salim S. Virani ${ }^{5} \cdot$ Zainab Samad $^{1,2,3}$
}

Accepted: 19 August 2021 / Published online: 23 October 2021

(c) The Author(s), under exclusive licence to Springer Science+Business Media, LLC, part of Springer Nature 2021

\begin{abstract}
Purpose of Review To revisit the importance of prevention strategies and policies in reducing the burden of ischemic heart disease in South Asian countries.

Recent Findings South Asia has seen rapid growth in its population with variable improvement in health indicators such as life expectancy at birth over the last three decades. Parallel to these improvements, there has been a stark rise in noncommunicable diseases (NCDs) but without a commensurate improvement in infrastructure/policies and health system interventions to address NCDs. South Asia is the epicenter of the cardiovascular disease (CVD) epidemic in Asia. It has a population that manifests accelerated atherosclerosis at a younger age. Poverty, lower health literacy, lack of health-promoting behaviors, poor urban design, rising air pollution, weak health systems, and lack and poor implementation of existing policies contribute to the continued rise in the incidence of CVD and the associated case fatality rates.

Summary A relatively young population presents an opportunity for implementation of prevention measures now which if not adequately utilized will result in an exponential rise in the CVD burden. There is a large gap between policymaking and implementation in this part of the world. Economic realities further constrain coverage of prevention policies; and therefore, stronger collaboration between governments, stakeholders, civil society, and regional and international funding agencies is needed to universally implement prevention strategies in South Asia.
\end{abstract}

Keywords Asia $\cdot$ South Asia $\cdot$ Health budget $\cdot$ Health policymaking $\cdot$ LMIC $\cdot$ Cardiovascular disease $\cdot$ Primary prevention . Secondary prevention

This article is part of the Topical Collection on Public Health Policy

Zainab Samad

samad.zainab@aku.edu

1 Department of Medicine, Aga Khan University, Karachi, Pakistan

2 Aga Khan University, Karachi, Pakistan

3 CITRIC Health Data Science Center, Aga Khan University, Karachi, Pakistan

4 Department of Community Health Sciences, Aga Khan University, Karachi, Pakistan

5 Department of Medicine, Baylor College of Medicine, Houston, USA

\section{Introduction}

Cardiovascular disease (CVD) is the leading cause of death worldwide with approximately 17.9 million or $31 \%$ of global deaths annually being attributable to it. Three-quarters of CVD-attributable deaths occur in low-to-middle-income countries (LMICs). Cardiovascular diseases (including ischemic heart disease (IHD), peripheral arterial disease (PAD), cerebrovascular disease, rheumatic heart disease, congenital heart disease, deep venous thrombosis, and pulmonary embolism) claim at least $30-35 \%$ of all-cause deaths each year in South Asia (SA) [1]. Most countries in Asia have higher age-adjusted mortality from CVD than Western countries [2]. The highest burden of CVD in Asia is centered around SA which also has higher rates of acute myocardial infarction (AMI) and at younger ages when compared with other regions of the world [3]. 
SA has seen changes in demography such as urbanization and industrialization which are known to affect CVD incidence. SA has witnessed a change in the urbanization rate from $16.7 \%$ in 1960 to $25 \%$ in 1990 , reaching $34.4 \%$ in 2019 [4]. These changes are accompanied by adverse behavior and lifestyle choices, physical inactivity, increasing rates of smoking, and poor dietary choices that are all implicated in the rising burden of CVD. However, strong policies addressing these risk factors in the vulnerable population have the potential for addressing CVD effectively in SA.

In this paper, we will review the CVD burden in SA countries as well as the existing global policies and guidelines and their implications to prevent and manage CVD. We will also propose ways in which new policies and related measures can be adapted to address the burden of CVD in South Asian countries.

\section{The Burden of Cardiovascular Diseases in South Asia}

Table 1 shows the population characteristics and burden of CVD and diabetes in 6 SA countries - Pakistan, India, Nepal, Bhutan, Sri Lanka, and Bangladesh [5-7]. SA has a current population of under two billion, which accounts for a quarter of the total world population [8]. For over 20 years, there has been a rise in deaths attributable to noncommunicable diseases, in particular cardiovascular diseases (IHD and stroke), with an IHD death rate as high as 152 to 204 per 100,000 of the population. A large segment of the population in SA countries is under the age of 40 years [5].

In SA, the age-standardized prevalence of IHD is 1500-1900/100,000 population, hypertensive heart disease is $70-90 / 100,000$ population, and ischemic stroke is 250-330/100,000 population [5]. Table 2 shows the countryspecific prevalence of various cardiovascular diseases in SA [9]. Among the highlighted six SA countries, Sri Lanka has the highest prevalence of IHD, closely followed by India, Bangladesh, and Pakistan. Sri Lanka also has the highest prevalence of atrial fibrillation and PAD in the region [9].

As of 2019, IHD accounted for $15.6 \%$ of total deaths in the SA region, followed by stroke ( $8.2 \%)$, hypertensive heart disease $(1.23 \%)$, and rheumatic heart disease (1.31\%). IHD accounted for $7.19 \%$ of total disability-adjusted life years (DALYs) in the SA region, followed by stroke (5.65\%). The burden of other CVD in terms of DALYs for SA is as follows: hypertensive heart disease $(0.85 \%)$, RHD $(0.42 \%)$, cardiomyopathy $(0.36 \%)$, atrial fibrillation $(0.33 \%)$ [10].

Although the total DALYs and deaths attributable to CVD rose globally between 1990 and 2019, the age-standardized rates for DALYs, deaths, and prevalent cases have declined, indicating that the global increase in CVD is mostly due to the population growth and aging. The Global Burden of

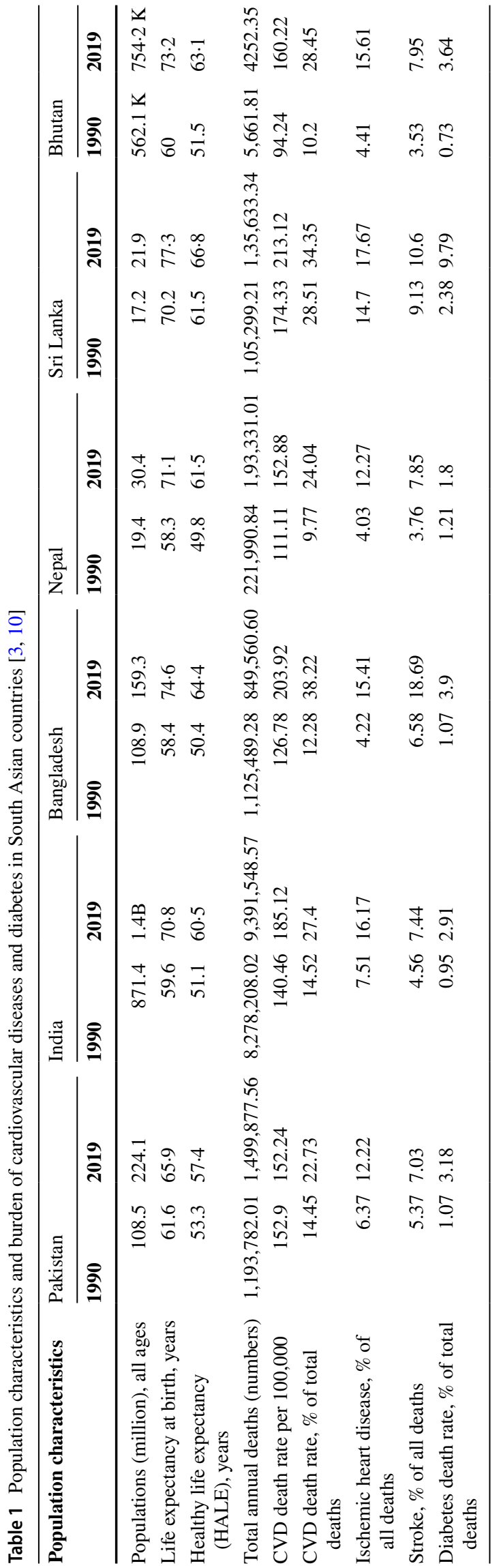


Table 2 Prevalence of cardiovascular disease in South Asian countries (per 100,000 of the population) [9]

\begin{tabular}{lccrrrr}
\hline CVD & Pakistan & India & Nepal & Bhutan & Sri Lanka & Bangladesh \\
\hline Atrial fibrillation and flutter & 87.13 & 239.84 & 214.67 & 220.07 & 416.07 & 210.31 \\
Cardiomyopathy and myocarditis & 12.05 & 7.65 & 12.84 & 16.73 & 33.37 & 14.01 \\
Cerebrovascular disease & 477.63 & 439.91 & 453.47 & 398.87 & 647.82 & 550.93 \\
Endocarditis & 0.66 & 0.68 & 0.64 & 0.75 & 0.96 & 0.66 \\
Hypertensive heart disease & 52.7 & 69.88 & 57.48 & 64.62 & 68.78 & 66.85 \\
Ischemic heart disease & 1137.29 & 1438.00 & 958.62 & 1038.01 & 1584.94 & 1152.40 \\
Peripheral arterial disease & 1365.3 & 1520.99 & 1536.24 & 1455.03 & 2396.45 & 1272.73 \\
\hline
\end{tabular}

Disease (GBD) 2019 study shows a rise in CVD death rates among all SA countries. Except for Nepal and Pakistan, CVD is the number one cause of death, and ischemic stroke is among the top ten causes of death in all SA countries [11].

\section{CVD Risk Factors in SA}

The increased susceptibility of SA to CVD can be attributable to the increased prevalence of traditional risk factors (Table 3) [7, 12]. Across the region, India has the highest prevalence of diabetes mellitus (DM). India also has the highest prevalence of physical inactivity, closely followed by Pakistan. Bangladesh has the highest prevalence of tobacco smoking, whereas Pakistan has the highest regional burden of obesity and raised total cholesterol. Fueled by rapid urbanization and consequent lifestyle changes, the increased prevalence of metabolic syndrome, against a background of genetic predisposition and predilection for inflammatory states, is thought to contribute to accelerated atherosclerosis in this part of the world [13].

While we await findings from the Mediators of Atherosclerosis in South Asians Living in America (MASALA) study that enrolled a cohort such as Multi-Ethnic Study of Atherosclerosis (MESA) to compare risk factors and outcomes across various ethnicities [14], results from INTERHEART indicate that the association of modifiable traditional risk factors with myocardial infarction in SA countries was no different from that in other ethnic groups [15]. The Prospective Urban Rural Epidemiology (PURE) study enrolled large epidemiological cohorts from 18 countries, including from South Asia, and estimated the prevalence of traditional risk factors among various world regions. This study showed higher case fatality rates for CVD in low- and low-middle-income countries (including SA) than the higher-income countries [16]. This trend is likely attributable to poor health literacy and poor health-system infrastructure lacking the required packages for dealing with CVD prevention and management. The comparison showed that the prevalence of DM, smoking, and physical inactivity was higher in SA when compared with that in North America and the European region. Subjects from SA were more likely to derive their daily energy from carbohydrates when compared with those from other regions [16]. This is likely a result of food choices driven mostly by cost and the need to gain satiety. Additionally, because of regional religious factors, many choose for an exclusively vegetarian diet. According to the PURE study, $65 \%$ of the study population derived at least $60 \%$ of energy from carbohydrates, and increased carbohydrate intake was associated with an overall increase in mortality, major cardiovascular disease event rate, and their composite [16, 17•]. These data suggest revisiting dietary recommendations in South Asia.

Table 4 shows the age-standardized summary exposure values (SEVs) for most detailed risk factors in SA countries [18•]. Exposure to smoking and a high-sodium diet is higher

Table 3 Prevalence of traditional risk factors for cardiovascular diseases in South Asian countries [7, 12]

\begin{tabular}{|c|c|c|c|c|c|}
\hline Risk factor & Pakistan & India & Sri Lanka & Bangladesh & Nepal \\
\hline Diabetes mellitus (prevalence $20-79$ y age), $2019^{*}$ & $8.8 \%$ & $10.4 \%$ & $7.9 \%$ & $9.2 \%$ & $7.2 \%$ \\
\hline $\begin{array}{l}\text { Physical inactivity (prevalence \% } 18 \text { y above) } \\
\text { (range)** }\end{array}$ & $33.7[20.3-47.0]$ & $34.0[22.3-47.7]$ & $28.9[21.3-37.9]$ & $27.8[16.4-39.1]$ & $13.4[11.2-15.6]$ \\
\hline Raised total cholesterol $\geq 5 \mathrm{mmol} / \mathrm{L}$ (range) $* *$ & $37.3[21.3-55.0]$ & $27.9[20.8-35.8]$ & $35.8[20.4-53.5]$ & 25.7 [13.4-41.9] & $23.2[11.8-38.1]$ \\
\hline $\begin{array}{l}\text { Obesity }(\mathrm{BMI} \geq 30, \text { age standardized estimate in \%) } \\
\text { (range) } * *\end{array}$ & $8.6[6.3-11.3]$ & $3.9[3.0-5.0]$ & $5.2[3.6-7.4]$ & $3.6[2.4-5.1]$ & $4.1[2.8-5.7]$ \\
\hline Smoking (range)** & $15.7[8.6-23.3]$ & $20.9[14.1-28.8]$ & $15[7.7-23.1]$ & $34.2[25.6-43.1]$ & $25.8[17.5-33.5]$ \\
\hline
\end{tabular}

*TW Bank. Diabetes Prevalence (2019)

**WH Organization. Global Health Observatory data repository 


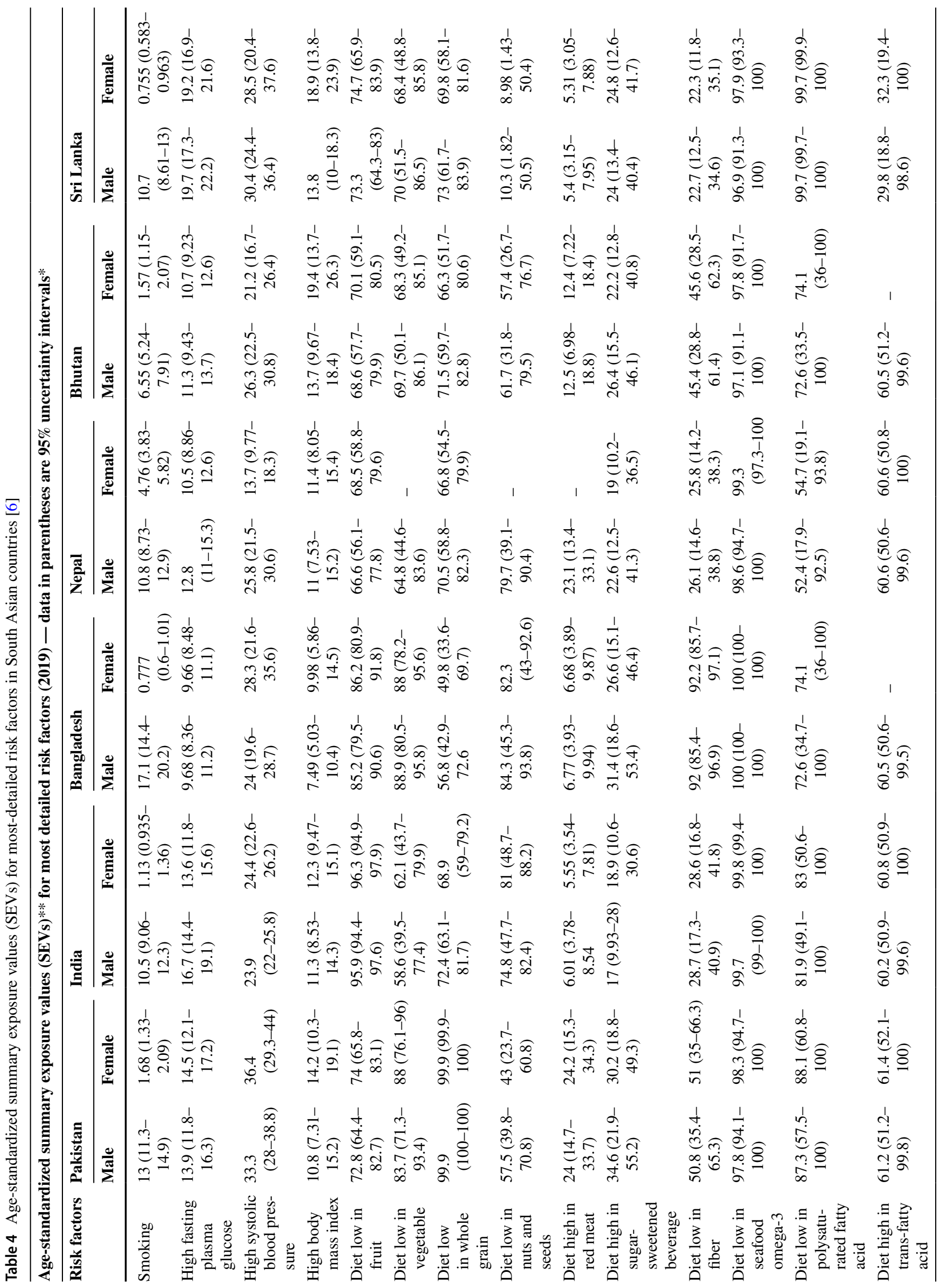


among SA men than that in SA women, whereas women have greater SEVs for a diet low in fresh fruits, and higher BMI and LDL levels than men. Across the entire region, the SEVs are high for a diet low in whole grains, a diet low in seafood omega-3, a diet low in poly-saturated fatty acid, a diet low in fresh fruits, vegetables, and nuts, and a diet high in trans-saturated fatty acids. Among all the six countries, Pakistan has the highest SEVs for raised systolic blood pressure and highest SEVs of a diet high in sugar-sweetened beverages [6].

Other risk factors that are relevant to IHD in SA include an overall greater lifetime exposure to inflammatory conditions (including acute infections, chronic granulomatous inflammations such as tuberculosis [19], and various rheumatologic disorders), increased rate of consanguineous marriages, and childhood malnutrition. Atherosclerosis has been recognized as a chronic low-grade inflammatory condition [20], and greater lifetime exposure to inflammatory conditions is likely to aggravate the rate of atherosclerosis in SA. A meta-analysis of 83,500 cases of tuberculosis showed that patients with tuberculosis are at an increased risk of developing IHD with a pooled risk ratio of 1.76 (95\% CI, 1.05-2.95; $I^{2}$ of $97 \%$ ) [21].

Genetic factors also contribute to the rising trend of CVD in SA. The relationship between consanguinity and the incidence of congenital heart disease is well known [22]. In some SA countries and cultures, the rate of consanguineous marriages is very high. For example, in Pakistan, almost $50 \%$ of marriages occur between first cousins [23]. This high rate of consanguinity has implications for monogenic and polygenic disorders and has been studied in a large prospective cohort. The genomic sequences of 10,503 individuals enrolled in the Pakistan Risk of Myocardial Infarction Study (PROMIS) were studied, and phenotypic consequences of "gene knockout" were analyzed. PROMIS participants had a fourfold higher median inbreeding coefficient (a measure of excess homozygosity) compared with outbred populations of European or African American ancestry [24]. The PROMIS cohort has established a higher prevalence of many loss-of-function mutations that could potentially increase or decrease the risk of CVD [25].

A high prevalence of childhood malnutrition is another important but often overlooked contributor to the risk of IHD in SA. A longitudinal study of individuals who were malnourished in the first year of life found that malnutrition was significantly associated with DM and metabolic syndrome, an important risk factor for IHD (adjusted OR $=4.8$ and 3.8, respectively; $p<0.05$ ) [26]. Childhood malnutrition is highly prevalent in SA countries. For example, one in three children under 5 (28.9\%) in Pakistan is underweight, and at least $9.5 \%$ of children are overweight [27]. At least $35.7 \%$ of the under-5 population are underweight in India [28]. Hence, the interplay of malnutrition leading to 
metabolic incompetence and metabolic syndrome later in life is very likely to potentiate the epidemiology of IHD in SA.

\section{Rheumatic Heart Disease}

Rheumatic heart disease (RHD) is one of the major CVD with a very high burden in SA. Second only to Oceania, SA carries the highest attributable DALYs for RHD (more than 400 DALYs per 100,000 persons) [29]. A community-based survey of 9430 subjects in a Pakistani city found a prevalence of 5.7 in 1000 [30]. Sriharibabu et al. [31] found the age-standardized RHD prevalence of 9.7/1000 population for RHD in 238 primary care centers of South India (44,164 subjects). Effective management of streptococcal throat infection is the key to preventing RHD. Financial limitations and poor health literacy potentiate the risk of RHD in the setting of untreated streptococcal sore throat infections. A large proportion of RHD in SA is diagnosed late in the disease stage and presents a challenge in terms of need for definite surgical or interventional care. Additionally, RHD remains the leading cause of CV morbidity in pregnancy in SA. Understanding the burden of RHD in SA and identification of risk factors and socioeconomic correlates is important to devise appropriate policies for the prevention strategies, but few surveillances or prevention programs exist in SA.

\section{Global Policies, Target, and Evidence of Tackling CVDs}

Evidence-informed healthcare policies have the potential to change the tide of the CVD epidemic globally and in South Asia. The real-world example of Finland demonstrates that with the right policies and comprehensive implementation, CVD rates can be reduced effectively. The North Karelia project in Finland instituted primary and secondary prevention strategies at a community level such as dietary advice, detection, management and follow-up for hypertension, and smoking cessation interventions. Following this, a major decline in smoking rates, serum cholesterol level, and blood pressure levels was seen [32]. In South Asia, where we are facing a large burden of CVD and associated risk factors, an age structure where a large segment of the population is under 40 represents a window of opportunity that must not be missed. For the purpose of this review, NCD policies are discussed for countries where CVD-specific policies are not separately available.

As per the report on Financing Global Health 2017, LMICs face a higher burden of NCDs (of which CVDs are the largest component) and a low percentage of total health spending. According to this report, NCDs accounted for
$67 \%$ of deaths in LMICs; however, only $2 \%$ of health funding was spent on the prevention of NCDs. A large portion (42\%) of NCD funding came through non-governmental organizations (NGOs) and foundations [33]. This disparity in funding between NCD burden and NCD-targeted health spending contributes to rising mortality and morbidity associated with CVD in this region. While the increasing burden of NCDs and inadequacy of current infrastructure for CVD is being recognized at the highest level including the development of NCD action plans, there is poor understanding of implementation plans across the SA.

The Global Action Plan 2013-2020 as proposed by WHO and adopted by a majority of SA countries aimed at reducing the burden of NCDs and prevalence of raised blood pressure (BP) by $25 \%$. This global plan called for the following principles to curb this burden: an evidence-based strategy, community empowerment and participation, interregional and international coordination, universal health coverage, an equity-based (rather than equality-based) approach, and a multisectoral approach. This global plan proposed a holistic approach to curb the burden and put down concrete goals for reducing CVD. These include a relative reduction (RR) of at least $25 \%$ in overall CVD mortality; a 10\% RR in alcohol consumption and physical inactivity; a 30\% RR in current smokers (age > 15 years); a $25 \%$ RR in raised BP; an RR of $30 \%$ in salt consumption, ensuring an $80 \%$ availability of medical amenities, drugs, and equipment in both private and public settings, ensuring that at least $50 \%$ of eligible patients receive medicines, counseling to prevent myocardial infarction and stroke, stopping the rise in obesity, and ensuring that at least $80 \%$ have access to essential medicines and equipment [34].

The equity-based approach recommended by the WHO is sensitive to the unequal distribution of socioeconomic determinants of CVD that affect the epidemiology in different economic strata of the world. For example, LMICs have a higher prevalence of physical inactivity and associated higher burden of hypertension, dyslipidemia, central obesity, and uncontrolled diabetes mellitus [35]. The WHO Framework Convention on Tobacco Control (FCTC) called for the regulation of tobacco products, limited interaction between tobacco industries and lawmakers, tobacco tax, ensuring health-warning labeling on tobacco products, ban of tobacco-product advertisement, increased public awareness, and introduction of addiction cessation programs [36]. Likewise, the Pakistan Dietary Guidelines for Better Nutrition (2018) is an example of how government should guide the daily nutritional requirement of individuals of all ages and groups [37]. Widespread knowledge and applicability of these national guidelines are essential.

Table 5 reviews current policies and plans for cardiovascular disease prevention in SA countries. 
Table 5 Current policies and plans for cardiovascular disease prevention in South Asian countries [37, 48-51]

\begin{tabular}{|c|c|c|c|}
\hline Country & Existing national-level policies/plans & $\begin{array}{l}\text { Integration of } \\
\text { CVD in primary } \\
\text { care }\end{array}$ & National plan for promoting physical activity \\
\hline Pakistan & $\begin{array}{l}\text { National Action Plan for Control of Non-communicable } \\
\text { Diseases and Health Promotion in Pakistan } \\
\text { WHO Framework Convention on Tobacco Control } \\
\text { (FCTC) (2005) (Pakistan became party) } \\
\text { Tobacco Control Cell and Pakistan Medical Research } \\
\text { Pakistan Hypertension League. Guideline for Detec- } \\
\text { tion, Control, and Management (1998) } \\
\text { Pakistan Dietary Guidelines for Better Nutrition } \\
\text { (2018) }\end{array}$ & No & No \\
\hline India & $\begin{array}{l}\text { National Multisectoral Action Plan for Prevention and } \\
\text { Control of Common Noncommunicable Diseases } \\
\text { Cigarettes and Other Tobacco Products Act (COTPA) } \\
\text { India Hypertension Control Initiative }\end{array}$ & Yes & No \\
\hline Bangladesh & $\begin{array}{l}\text { National Non-communicable Disease Control (Janu- } \\
\text { ary 2017-June 2022) }\end{array}$ & Yes & National Sports Policy (1998) \\
\hline Nepal & $\begin{array}{l}\text { Multisectoral Action Plan for the Prevention and Con- } \\
\text { trol of Non-communicable Diseases (2014-2020) }\end{array}$ & No & No \\
\hline Sri Lanka & $\begin{array}{l}\text { National Multisectoral Action Plan for the Preven- } \\
\text { tion and Control of Noncommunicable Diseases } \\
(2016-2020)\end{array}$ & Yes & $\begin{array}{l}\text { The Physical Activity Guidelines for Sri Lankans } \\
\text { (2018) }\end{array}$ \\
\hline Bhutan & $\begin{array}{l}\text { The Multisectoral National Action Plan for the Pre- } \\
\text { vention and Control of Noncommunicable Diseases } \\
(2015-2020)\end{array}$ & Yes & National Health Promotion Strategic Plan (2015-2023) \\
\hline
\end{tabular}

\section{Implications of the Health Economy and Resource Allocation in SA}

The South Asian block of countries generally lands in the LMIC group. On average, the national net income ranges from US $\$ 919$ to 3671 . Table 6 highlights the current health-budget allocation of few SA countries [38]. Sri Lanka has the highest current health expenditure per capita, followed by Bhutan, India, Nepal, Pakistan, and Bangladesh. Sri Lanka also has the highest net national income per capita. The current health expenditure of SA countries (\% of GDP) ranges from 2.34 to $5.84 \%$ which is comparable to that of Turkey (4.7\%) and Mexico (5.5\%) but lower than that of Iran (8.66\%) and far lower than that of the USA (17\%) and UK (10.3\%) [39]. Budget allocation in SA countries needs to be increased for achieving universal health coverage and providing therapies for preventing and reducing case fatality rates.

The WHO has proposed various models for scaling up implementation strategies against NCDs that span approximately 5 years with different phases such as planning, policy development, and partial and complete implementation [40]. One of such models employs the principle of identifying the high-risk population and using a target-population strategy. It provides a plan to estimate the cost burden based on the target population. The model focuses on reducing tobacco consumption, treating diabetes mellitus, and promoting healthy eating and physical activity. This model defined tobacco control steps: modeled alcohol control strategies included banning advertisements, reducing access to retailed alcohol,

Table 6 Health budget expenditure in a few South Asian countries [38]

\begin{tabular}{|c|c|c|c|c|c|c|}
\hline Year 2018 & Pakistan & India & Bangladesh & Nepal & Bhutan & Sri Lanka \\
\hline Adjusted net national income per capita (US\$) & 1469 & 1735 & 1619 & 919 & 2641 & 3671 \\
\hline Current health expenditure (\% of GDP) & 3.2 & 3.54 & 2.34 & 5.84 & 3.06 & 3.76 \\
\hline Current health expenditure per capita (current US\$) & 42.87 & 72.83 & 41.91 & 57.85 & 102.74 & 157.47 \\
\hline Domestic private health expenditure per capita, PPP (current international \$) & 113.83 & 199.06 & 83.88 & 118.82 & 46.38 & 292.94 \\
\hline $\begin{array}{l}\text { Domestic general government health expenditure per capita, PPP (current } \\
\text { international \$) }\end{array}$ & 63.34 & 74.16 & 18.62 & 45.19 & 256.23 & 212.41 \\
\hline
\end{tabular}


raised taxes, mass media education, and individual meetings; and diet and physical activity strategies focused on reducing salt intake, replacing trans-fat with poly-unsaturated fat, and promoting public awareness about primary and secondary prevention. The model predicted the costs of interventions (annual cost per person in US\$) in LMICs. The total estimated cost of various interventions to reduce four basic risk factors for CVD in LMICs was calculated. This estimation concluded that the median per-person cost of intervention in CVD prevention in LMICs setting is less than \$0.20 when compared to a cost of $\$ 0.50$ in upper-middle-income countries. This model has predicted a per-person annual cost of combatting NCDs to be $\$ 74$ (including lifestyle changes and medical therapy). However, according to this estimate, the cost of lifestyle changes was far less than the medical therapy [40]. The current per capita health expenditures for Pakistan, India, Bangladesh, Nepal, Bhutan, and Sri Lanka are US\$ 43, 73, 42, 58, 103, and 157, respectively. This estimation may help health policymakers and economists for appropriate budget allocation in SA countries.

In the first Global Ministerial Conference on Healthy Lifestyles and Noncommunicable Disease Control held in April 2011, WHO urged all ministers to reassess and project healthcare situations in their countries based on the change in demographics and respective allocation of healthcare workers. In SA countries which face a chronic shortage of medical professionals, models of care that utilize community healthcare workers may be a cost-effective strategy for population-level prevention. For example, the Control of Blood Pressure and Risk Attenuation in Bangladesh, Pakistan, and Sri Lanka (COBRA-BPS) [41••] study showed that in rural communities, a cost-effective home-based intervention utilizing community health workers for hypertension led to a greater reduction in $\mathrm{BP}(53.2 \%)$ than the usual care $(43.7 \%)$ (relative risk, 1.22; 95\% CI, 1.10-1.35). Hence, it is imperative that policymakers, stakeholders, and public-private partners are aware of cost-effective strategies for reducing the burden of CVD in the SA region.

\section{Curbing the Burden of CVD in South Asia - Evidence-Informed Strategies for Crafting a Way Forward}

The strategies to address the burden of CVD in SA can broadly be divided into health sector interventions and intersectoral interventions, but all require policy and governmental enforcement:

\section{Health Sector Interventions}

The health sector bears the primary responsibility for health burden estimation, deployment of appropriate administrative and medical staff, and ensuring coordinated care among all levels of care. A poorly funded fragmented health system represents a significant problem, and this is also compounded by an inadequate healthcare workforce in SA [42].

Advisable interventions include:

- Ensuring medical supplies and the provision of basic medical equipment and medicines at all levels of care is imperative. An increase in induction of qualified medical staff is required to increase the doctor-patient or nursepatient ratio in SA. Additionally, retraining the existing workforce is essential to tackle CVD.

- At the primary care level, the traditional focus on maternal/child health and communicable disease must be expanded to include NCDs. Considering the large burden of CVD in this region, CVD prevention should be integrated into the primary healthcare programs and exclusive budgets should be allocated for CVD prevention.

- Annual screening or surveillance programs for cardiovascular risk assessment including screening for hypertension, DM, dyslipidemia, and obesity should be ensured at all primary and secondary healthcare facilities.

- Low-cost health screening packages must be introduced for the public and especially underprivileged populations that cannot access hospitals and health facilities where prevention packages are offered doorstep at the community level.

- Universal health coverage (UHC) should be ensured. This would ensure regular health checkups of masses and identify diseases at an early stage. Healthcare models that focus on health which include preventive visits should be adopted.

- There is a need for a coordinated system of care for CVD whereby all regional centers work in a coordinated fashion and follow appropriate referral pathways to ensure timely interventions for CVD emergencies such as acute myocardial infarction and stroke. Such coordinated systems of care need to be enforced and championed by the government.

\section{Inter-sectoral Interventions}

The work and action of multiple sectors outside the health sector contribute to the risk of CVD. These include the food industry, workplace, the education system, and the role of media. Multi-sectoral policy changes are also required to affect CVD incidence.

Awareness plays a pivotal role in CVD prevention [43]; and therefore, it is imperative that mass public awareness is created, sustained, and reinforced in a timely fashion. A mass media campaign in Vietnam focusing on reducing salt intake and tobacco smoking, and avoiding a high-fat diet was found to be very cost-effective to reduce blood pressures, 
followed by individual advice on drug therapy for hypertension [44]. Policymaking for CVD prevention needs to cover multiple aspects such as food labeling and advertisement, food tax and subsidies, tobacco advertisement, worksite prevention, workplace, and school annual medical assessments, and critically evaluate marketing standards of food products. It is imperative that the importance of physical activity is disseminated and encouraged through built spaces and planned work, home, and urban environments and through the availability of excellent public transportation.

Singh et al. evaluated the cost-effectiveness of interventions to control IHD and DM in SA, but due to significant differences in policy frameworks across the region, the analysis was limited. However, the majority of public health measures such as tobacco and alcohol taxation, universal screening for IHD, preconception care for women of reproductive age, individual-based treatment and prevention for hypertension, stroke and heart failure, and combination therapy were found to be cost-saving [45].

Additional population-level interventions include:

- Promoting physical activity through a variety of measures including mandatory physical activity sessions in educational institutes; creating segregated public spaces for exercise considering sociocultural aspects; reinforcing the need for physical inactivity at every medical or institutional encounter; and promoting health benefits of exercise as a media reminder during advertisement gaps.

- Instituting primary prevention measures at educational institutions and public/private workplaces. These include annual health checks, mandatory physical activity, and health promotion campaigns.

- Gender-sensitive prevention interventions should be ensured given the differential burden of risk factors in men and women. Physical activity and obesity rates are higher in women, whereas smoking and hypertension are higher among men.

- Restriction on advertisement of fast food and sugary drinks, promotion of economic agricultural reforms, and provision of exhibition opportunities to agricultural vendors can all help increase awareness of public regarding the health benefits of natural food and avoidance of unhealthy "junk-food."

- Public-private partnership is essential to ensure the quality and outcomes of prevention interventions. Strict accountability at the grass-roots level with short-term targets should be set focusing on small villages, tehsil, cities, and so on.

- Increasing the price and instituting tobacco tax; enforcing policies to restrict indoor and public place smoking; banning media advertisement of tobacco and tobacco products; and mass rehabilitation programs for tobacco addiction.
- Appropriate labeling of foods with nutrients and calories would help consumers make informed choices based on their health conditions. Additionally, food packages should show important warnings and information about the storage of food.

- Sugar tax policies can help reduce the consumption of table sugar which overwhelmingly affects the rates of metabolic syndrome and DM in the region.

\section{COVID-19 Pandemic and CVD Prevention}

The COVID-19 pandemic has affected the trajectory of CVD in SA, and the full implications of COVID-19 on the CVD burden will be only realized with time. COVID19-related lockdowns and work-from-home policies have led to increased sedentary behavior. Medical care of common CVD risk conditions such as hypertension and diabetes has been disrupted due to either patient fear of visiting medical facilities or lockdown-related closure of medical facilities. Likewise, emergency care of cardiovascular conditions has also been affected with poorer outcomes and the consequent impact on survival and quality of life. A WHO survey evaluated the delivery of NCD services during the pandemic, and according to the results, a total of 122 countries reported disruption of NCD services such as hypertension and diabetes management and cardiovascular emergencies. According to the report, $61 \%$ of LMICs (including SA countries) included continuity of NCD services in national COVID-19 plans compared with $78 \%$ in high-income countries [46]. The economic burden of the pandemic has disrupted the management of CVD overall. Additionally, COVID-19 illness itself can cause a wide range of CVD. There might be a need now to incorporate COVID-19 packages into the NCD package for CVD prevention and management; and hence, there is a need for this preparedness among regional policymakers.

\section{Conclusion}

Prevention strategies are cost-effective yet underrated tools to combat CVD. There is a need to reallocate the health budget and revise healthcare policies to house the merited role of prevention strategies. Cost-effective and cultural-geographical friendly policymaking is the first step to curb the burgeoning burden of CVD in SA countries.

Author Contribution PS wrote the manuscript; MH, SK, and AH contributed to the literature search and data presentation; SM, SV, and SS reviewed critically and edited the manuscript; ZS supervised from conceptualization to final editing of the manuscript. 


\section{Compliance with Ethical Standards}

Conflict of Interest SV: Department of Veterans Affairs, Tahir and Jooma Family, World Heart Federation Honorarium: American College of Cardiology (Associate Editor for Innovations, acc.org). The other authors declare that they have no conflict of interest.

Human and Animal Rights and Informed Consent This article does not contain any studies with human or animal subjects performed by any of the authors.

\section{References}

Papers of particular interest, published recently, have been highlighted as:

- Of importance

$\bullet$ Of major importance

1. Organization WH. Non-communicable diseases and mental health in South Asia region. 2021.

2. Ohira T, Iso H. Cardiovascular disease epidemiology in Asia: an overview. Circ J. 2013;77(7):1646-52.

3. Joshi P, Islam S, Pais P, Reddy S, Dorairaj P, Kazmi K, et al. Risk factors for early myocardial infarction in South Asians compared with individuals in other countries. JAMA. 2007;297(3):286-94.

4. Bank TW. United Nations Population Division. World urbanization prospects: 2018

5. Wang $\mathrm{H}$, Abbas KM, Abbasifard M, Abbasi-Kangevari M, Abbastabar H, Abd-Allah F, et al. Global age-sex-specific fertility mortality, healthy life expectancy (HALE), and population estimates in 204 countries and territories, 1950-2019: a comprehensive demographic analysis for the Global Burden of Disease Study 2019. Lancet (London, England). 2020;396(10258):1160-203.

6. Kyu HH, Abate D, Abate KH, Abay SM, Abbafati C, Abbasi N, et al. Global, regional, and national disability-adjusted life-years (DALYs) for 359 diseases and injuries and healthy life expectancy (HALE) for 195 countries and territories, 1990-2017: a systematic analysis for the Global Burden of Disease Study 2017. Lancet (London, England). 2018;392(10159):1859-922.

7. Organization WH. Global Health Observatory data repository. 2021.

8. Worldometer. World population by region. 2021.

9. Roth GA, Johnson C, Abajobir A, Abd-Allah F, Abera SF, Abyu $\mathrm{G}$, et al. Global, regional, and national burden of cardiovascular diseases for 10 causes, 1990 to 2015. J Am Coll Cardiol. 2017;70(1):1-25.

10. (IHME) TIfHMaE. Global burden of diseases. 2019.

11. IHME. GBD 2019 Cause and risk summaries. 2019.

12. Bank TW. Diabetes prevalence. 2019.

13. Volgman AS, Palaniappan LS, Aggarwal NT, Gupta M, Khandelwal A, Krishnan AV, et al. Atherosclerotic cardiovascular disease in South Asians in the United States: epidemiology, risk factors, and treatments: a scientific statement from the American Heart Association. Circulation. 2018;138(1):e1-34.

14. Kanaya AM, Kandula N, Herrington D, Budoff MJ, Hulley $\mathrm{S}$, Vittinghoff E, et al. Mediators of Atherosclerosis in South Asians Living in America (MASALA) study: objectives, methods, and cohort description. Clin Cardiol. 2013;36(12):713-20.

15. Yusuf S, Hawken S, Ounpuu S, Dans T, Avezum A, Lanas F, et al. Effect of potentially modifiable risk factors associated with myocardial infarction in 52 countries (the INTERHEART study): case-control study. Lancet (London, England). 2004;364(9438):937-52.

16. Dehghan M, Mente A, Zhang X, Swaminathan S, Li W, Mohan $\mathrm{V}$, et al. Associations of fats and carbohydrate intake with cardiovascular disease and mortality in 18 countries from five continents (PURE): a prospective cohort study. Lancet (London, England). 2017;390(10107):2050-62.

17. Swaminathan S, Dehghan M, Raj JM, Thomas T, Rangarajan $\mathrm{S}$, Jenkins D, et al. Associations of cereal grains intake with cardiovascular disease and mortality across 21 countries in Prospective Urban and Rural Epidemiology study: prospective cohort study. BMJ. 2021;372:m4948. This analysis based on a large number of subjects showed that higher intake of refined grains was associated with higher mortality and cardiovascular events, globally.

18. Murray CJL, Aravkin AY, Zheng P, Abbafati C, Abbas KM, Abbasi-Kangevari M, et al. Global burden of 87 risk factors in 204 countries and territories, 1990-2019: a systematic analysis for the Global Burden of Disease Study 2019. Lancet. 2020;396(10258):1223-49. Hypertension followed by smoking was the leading level 2 risk factor globally for attributable deaths. The paper highlights the importance of smoking related regulation and policymaking in reducing the high attributable death burden.

19. Huaman MA, Henson D, Ticona E, Sterling TR, Garvy BA. Tuberculosis and cardiovascular disease: linking the epidemics. Trop Dis Travel Med Vaccines. 2015;1.

20. Wong BW, Meredith A, Lin D, McManus BM. The biological role of inflammation in atherosclerosis. Can J Cardiol. 2012;28(6):631-41.

21. Wongtrakul W, Charoenngam N, Ungprasert P. Tuberculosis and risk of coronary heart disease: a systematic review and meta-analysis. Indian J Tuberc. 2020;67(2):182-8.

22. Ramegowda S, Ramachandra NB. Parental consanguinity increases congenital heart diseases in South India. Ann Hum Biol. 2006;33(5-6):519-28.

23. Studies. NIoP. Demographic and health survey. 2017-18.

24. Saleheen D, Zaidi M, Rasheed A, Ahmad U, Hakeem A, Murtaza M, et al. The Pakistan Risk of Myocardial Infarction Study: a resource for the study of genetic, lifestyle and other determinants of myocardial infarction in South Asia. Eur J Epidemiol. 2009;24(6):329-38.

25. Saleheen D, Natarajan P, Armean IM, Zhao W, Rasheed A, Khetarpal SA, et al. Human knockouts and phenotypic analysis in a cohort with a high rate of consanguinity. Nature. 2017:544(7649):235-9.

26. Fischer L, Galler J. Early childhood malnutrition increases metabolic syndrome in adulthood. FASEB J. 2015;29(S1):258-62.

27. Organziation WH. National Nutrition Survey Pakistan 2018. 2018.

28. Murarkar S, Gothankar J, Doke P, Pore P, Lalwani S, Dhumale $\mathrm{G}$, et al. Prevalence and determinants of undernutrition among under-five children residing in urban slums and rural area, Maharashtra, India: a community-based cross-sectional study. BMC Public Health. 2020;20(1):1559.

29. Roth Gregory A, Mensah George A, Johnson Catherine O, Addolorato G, Ammirati E, Baddour Larry M, et al. Global burden of cardiovascular diseases and risk factors, 1990-2019. J Am Coll Cardiol. 2020;76(25):2982-3021.

30. Rizvi SF, Khan MA, Kundi A, Marsh DR, Samad A, Pasha O. Status of rheumatic heart disease in rural Pakistan. Heart. 2004;90(4):394-9.

31. Sriharibabu M, Himabindu Y, Kabir Z. Rheumatic heart disease in rural South India: a clinico-observational study. J Cardiovasc Dis Res. 2013;4(1):25-9. 
32. Vartiainen E. The North Karelia project: cardiovascular disease prevention in Finland. Glob Cardiol Sci Practice. 2018;2018(2):13.

33. IHME GBoD. High burden, low budget: non-communicable diseases in low- and middle-income countries. 2018.

34. Organization WH. WHO tools to prevent and control noncommunicable diseases. 2021.

35. Organization WH. World statistics. 2019.

36. FCTC abtCotPttW. WHO Framework Convention on Tobacco Control. 2003.

37. Ministry of Planning Development and Reform. Pakistan Dietary Guidelines for Better Nutrition. 2018.

38. Bank TW. External health expenditure per capita, PPP (current international \$). 2011.

39. OECD.stat. Health expenditure and funding. 2021.

40. Organization WH. Scaling up action against NCDs: how much will it cost. 2011.

41.• Jafar TH, Gandhi M, de Silva HA, Jehan I, Naheed A, Finkelstein EA, et al. A community-based intervention for managing hypertension in Rural South Asia. N Engl J Med. 2020;382(8):717-26. This study is an excellent example of how a community-based and cost-effective intervention of home visits can help achieve significant blood pressure control in South Asian countries.

42. Organization WH. The labour market for human resources for health in lowand middle-income countries. 2012.

43. Koniak-Griffin D, Brecht ML. Awareness of cardiovascular disease and preventive behaviors among overweight immigrant Latinas. J Cardiovasc Nurs. 2015;30(5):447-55.
44. Ha DA, Chisholm D. Cost-effectiveness analysis of interventions to prevent cardiovascular disease in Vietnam. Health Policy Plan. 2011;26(3):210-22.

45. Singh K, Chandrasekaran AM, Bhaumik S, Chattopadhyay K, Gamage AU, Silva PD, et al. Cost-effectiveness of interventions to control cardiovascular diseases and diabetes mellitus in South Asia: a systematic review. BMJ Open. 2018;8(4):e017809.

46. Organization WH. Rapid assessment of servcie delivery of noncommunicable diseases during the COVID-19 pandemic. 2020.

47. IHME. Health data term definitions. 2021.

48. Nishtar S, Faruqui AM, Mattu MA, Mohamud KB, Ahmed A. The national action plan for the prevention and control of noncommunicable diseases and health promotion in Pakistancardiovascular diseases. J Pak Med Assoc. 2004;54(12 Suppl 3):S14-25

49. UNION T. Tobacco control in Pakistan. Published on 12 August 2020 updated: 2 October 2020. Authored by Anonymous. THE TOBACCO EPIDEMIC.

50. Ministry of Health and Family Welfare GoI. Prevention and control of common non-communicable disease. 2017.

51. Misra A, Tandon N, Ebrahim S, Sattar N, Alam D, Shrivastava U, et al. Diabetes, cardiovascular disease, and chronic kidney disease in South Asia: current status and future directions. BMJ (Clinical research ed). 2017;357:j1420.

Publisher's Note Springer Nature remains neutral with regard to jurisdictional claims in published maps and institutional affiliations. 